\title{
Timing of appropriate empirical antimicrobial administration and outcome of adults with community-onset bacteremia
}

\author{
Ching-Chi Lee ${ }^{1,2,3} \mathbb{D}$, Chung-Hsun Lee ${ }^{4,5}$, Ming-Yuan Hong ${ }^{4,5}$, Hung-Jen Tang ${ }^{6,7,9^{*}}$ and Wen-Chien Ko ${ }^{3,4,8^{*}}$
}

\begin{abstract}
Background: Early administration of appropriate antimicrobials has been correlated with a better prognosis in patients with bacteremia, but the optimum timing of early antibiotic administration as one of the resuscitation strategies for severe bacterial infections remains unclear.

Methods: In a retrospective cohort study, adults with community-onset bacteremia at the emergency department (ED) were analyzed. Effects of different cutoffs of time to appropriate antibiotic (TtAa) administration after arrival at the ED on 28-day mortality were examined, after adjustment for independent predictors of mortality identified by multivariate regression analysis.

Results: Among 2349 patients, the mean (interquartile range) TtAa was $2.0(<1$ to 12) hours. All selected cutoffs of TtAa, ranging from 1 to 96 hours, were significantly associated with 28-day mortality (adjusted odds ratio (AOR), 0.54-0.65, all $P<0.001$ ), after adjustment of the following prognostic factors: fatal comorbidities (McCabe classification), critical illness (Pitt bacteremia score (PBS) $\geq 4$ ) on arrival at the ED, polymicrobial bacteremia, extended-spectrum betalactamase-producer bacteremia, underlying malignancies or liver cirrhosis, and bacteremia caused by pneumonia or urinary tract infections. The adverse impact of TtAa on 28-day mortality was most evident at the cutoff of 48 hours, as the lowest AOR was identified $(0.54, P<0.001)$. In subgroup analyses, the most evident TtAa cutoff (i.e., the lowest AOR) remained at 48 hours in mildly ill (PBS $=0$; $A O R \quad 0.47 ; P=0.04$ ) and moderately ill (PBS $=1-3$; $A O R \quad 0.55 ; P=0.02)$ patients, but shifted to 1 hour in critically ill patients (PBS $\geq 4$; AOR 0.56; $P<0.001$ ).

Conclusions: The time from triage to administration of appropriate antimicrobials is one of the primary determinants of mortality. The optimum timing of appropriate antimicrobial administration is the first 48 hours after non-critically ill patients arrive at the ED. As bacteremia severity increases, effective antimicrobial therapy should be empirically prescribed within 1 hour after critically ill patients arrive at the ED.
\end{abstract}

Keywords: Initial antibiotic therapy, Inappropriateness, Bloodstream infection, Prognosis

\section{Background}

Despite improvements in hemodynamic support and antibiotic therapy, bacteremia is still associated with high morbidity and mortality, which contribute substantially to healthcare costs [1]. Community-onset bacteremia, with an annual incidence of $0.82 \%$ in a population-based study [2], is a common problem for clinicians. In addition to early establishment of vascular

\footnotetext{
* Correspondence: 8409d1@gmail.com; winston3415@gmail.com ${ }^{6}$ Department of Medicine, Chi-Mei Medical Center, Tainan, Taiwan ${ }^{3}$ Department of Internal Medicine, National Cheng Kung University Hospital, Tainan, Taiwan

Full list of author information is available at the end of the article
}

access, fluid resuscitation, and removal of infection source, particularly in critically ill patients, antimicrobial therapy has been regarded as one of the mainstays of bacteremia treatment [3]. Although most investigations have concluded that early administration of appropriate antimicrobials could be linked to a favorable prognosis in the individuals with bacteremia [4-7], several studies have not identified the survival benefit of early appropriate antimicrobial therapy $[8,9]$. Furthermore, the timing of the first dose of appropriate antibiotics remains a controversial point, and thus appropriate empirical antimicrobial therapy has been defined as the interval 
between bacteremia onset and antimicrobial administration, ranging widely from $<24$ hours to $<48$ hours in previous reports $[7,10,11]$. To our knowledge, the Surviving Sepsis Campaign (SCC) "International guidelines for the management of severe sepsis and septic shock" recommend that an appropriate antimicrobial therapy be administered within the first 1 hour of recognition of severe sepsis or septic shock [3]. However, focusing on patients with bacteremia, the question "what priority is early enough for appropriate antibiotic administration?" has been debated. Therefore, to describe the beneficial effects of appropriate empirical antimicrobial administration on clinical outcome and to further achieve the "optimum timing" of appropriate antimicrobial administration, we compared the outcome impact of different time cutoffs from emergence department (ED) triage to appropriate antimicrobial administration for adults with community-onset bacteremia.

\section{Methods}

\section{Study design}

This retrospective cohort study was conducted from January 2008 to December 2013 at the ED of a medical center in southern Taiwan. The study hospital is a 1200bed, university-affiliated medical center with an annual ED census of approximate 70,000 patients. The study was reported by the format recommended by the Strengthening the Reporting of Observational Studies in Epidemiology (STROBE) statement; partial clinical information on this study cohort has been published [10-13].

During the study period, the blood cultures sampled at the ED were screened for bacterial growth by a computer database. Clinical information on adults with bacteremia was retrieved from medical charts. In the patients with multiple episodes of bacteremia, only the first episode of each patient was included. Patients were excluded if they had hospital-onset bacteremia, contaminated blood cultures, bacteremia diagnosed prior to visiting the $E D$, inappropriate dosage or route of antimicrobial administration during all hospitalization, or incomplete clinical information.

\section{Data collection}

A predetermined form was used to collect demographic and clinical characteristics, including age, vital signs on arrival at the ED, comorbidities, type of antimicrobial agents administered and duration of administration, bacteremia source, length of hospital stay, bacteremia severity (Pitt bacteremia score (PBS)) on arrival at the ED, comorbidity severity (McCabe classification), and patient outcome. Of all eligible patients, information was retrieved by retrospective review of medical charts, which was done by two authors, and any discrepancy was resolved by discussion between the authors. The primary endpoint was crude mortality within 28 days after onset of bacteremia (arrival at the ED).

\section{Definitions}

Community-onset bacteremia indicates that the place of onset of the episode of bacteremia is the community, which includes long-term healthcare facility-acquired and community-acquired bacteremia, as previously described [11, 14]. Polymicrobial bacteremia was defined as the isolation of more than one microbial species from a single bacteremia episode. Blood culture samples with potentially contaminating pathogens were considered to be contaminated in accordance with the previous criteria [15].

As previously described [10, 11], the antimicrobial therapy was considered appropriate when all the following criteria were fulfilled: (1) the route and dosage of antimicrobial agents were administered as recommended in the Sanford Guide [16] and (2) the bacteremia pathogens were susceptible in vitro to the administrated antimicrobial agent based on the contemporary breakpoints of the Clinical and Laboratory Standards Institute (CLSI) [17]. The time to appropriate antibiotic (TtAa) measured in hours was defined as the period between the arrival at the ED (i.e., ED triage) and the administration of appropriate antimicrobials [18]. The bacteremia severity was graded according to the PBS using a previously validated scoring system based on vital signs, use of inotropic agents, mental status, receipt of mechanical ventilation, and cardiac arrest $[11,14]$. Comorbidities were defined as described previously [19] and malignancies included hematological malignancies and solid tumors. The prognosis of preexisting diseases was assessed by a previous delineated classification system (McCabe classification) [20]. Bacteremia sources were determined clinically on the basis of the previous definitions [11]. Crude mortality was used to define death from all causes.

\section{Microbiological methods}

Blood cultures were incubated in a BACTEC 9240 instrument (Becton Dickinson Diagnostic Systems, Sparks, $\mathrm{MD}$, USA) for 5 days at $35{ }^{\circ} \mathrm{C}$. Bacteremia aerobic isolates were prospectively collected during the study period. The bacterial species was identified by means of the Vitek 2 system (bioMérieux, Durham, NC, USA), and antimicrobial susceptibility was determined by the disk diffusion method, based on the performance standards of CLSI in 2016 [17]. The tested drugs included ampicillin/sulbactam, piperacillin/tazobactam, cefazolin, cefuroxime, cefotaxime, ceftazidime, cefepime, ertapenem, imipenem, and levofloxacin. If the patient was empirically treated by other agents, the susceptibility of the indicated agent was measured. For Escherichia coli, Klebsiella species, and Proteus mirabilis (EKP), extended-spectrum 
beta-lactamase (ESBL) production was detected by the phenotypic confirmatory test with the cephalosporinclavulanate combination disks recommended by the previous CLSI guidelines in 2009 [21].

\section{Statistical analyses}

Statistical analyses were performed using the Statistical Package for the Social Sciences for Windows (Version 20.0; Chicago, IL, USA). Clinical data, demographic data, severity, and patient outcomes were compared using the Fisher exact test or the Pearson chi-square test for categorical variables and the independent samples $t$ test or the Mann-Whitney $U$ test for continuous variables. To assess the independent predictors with adjusted odds ratios (AORs), the variables in the univariate analysis with a $P$ value $<0.05$ were included in a stepwise and backward multivariable logistic regression model. Several TtAa cutoffs were selected: 1 hour, 6 hours, 12 hours, 24 hours, 48 hours, 72 hours, and 96 hours. Cox regression was used to study the impact of varied TtAa cutoffs on clinical outcomes after adjustment for independent risk factors for 28-day mortality.

As all available data were used to maximize the power, no sample size calculation was performed. As suggested, at least $8-10$ events per variable are required for reliable multiple logistic regression analysis [22]. For missing data, a complete case analysis was conducted if the percentage of missing values was $<5 \%$. If the percentage of missing values was $\geq 5 \%$, appropriate imputation was performed [23]. A two-tailed $P$ value $<0.05$ was considered significant.

\section{Results}

\section{Demographics and clinical characteristics of the patient} cohort

Of 3934 patients with bacterial growth in blood cultures, 2349 adults with community-onset bacteremia were analyzed, after exclusion of 1503 adults with contaminated blood cultures, 66 with hospital-onset bacteremia or with bacteremia prior to arrival at the ED, 13 with an uncertain outcome within 28 days of onset of bacteremia, and 3 with inappropriate dosage or route of antimicrobial administration during all hospitalization. Among the 2349 eligible patients the mean age was 67.9 years and 1029 patients (51.5\%) were male. The ten most common sources of bacteremia were urinary tract infections (748 patients, 31.1\%), pneumonia (325 patients, 13.5\%), intra-abdominal infections (312 patients, $13.0 \%$ ), skin and soft-tissue infections (244 patients, $10.1 \%)$, biliary tract infections (220 patients, 9.2\%), primary bacteremia (191 patients, 7.9\%), bone and joint infections (92 patients, 3.8\%), vascular-line infections (90 patients, 3.7\%), liver abscess (79 patients, 3.3\%), and infective endocarditis (71 patients, $3.0 \%$ ).
The mean (interquartile range) length of ED stay was 19.6 (5.3-25.6) hours. Among the patients, 1829 (77.9\%) were admitted to general wards and 324 (13.8\%) to intensive care units (ICUs). The mean (interquartile range) length of hospital stay was 14.9 (6.1-17.0) days. There were 77 patients (3.3\%) who died during the ED stay. There were 119 (5.1\%) patients discharged from the ED and followed up in the outpatient clinics. The proportion of critically ill $(\mathrm{PBS} \geq 4)$ and mildly ill $(\mathrm{PBS}=0)$ patients at ED arrival was 20.3\% (476 patients) and 26.9\% (633), respectively. The overall 28 -day crude mortality rate was $14.5 \%$ (340 patients).

\section{Baseline characteristics of fatal and surviving patients}

Univariate analyses were used to compare clinical variables among adults with fatal and surviving outcome (Table 1). Nursing-home residents, male gender, critical illness (PBS $\geq 4$ ) on arrival at the ED, initial syndromes of severe sepsis or septic shock on arrival at the ED, fatal comorbidities (McCabe classification), polymicrobial bacteremia, K. pneumoniae, Pseudomonas species, or ESBL-producing EKP bacteremia, bacteremia pneumonia, underlying neurological diseases or liver cirrhosis, thrombocytopenia, or elevated serum levels of Creactive protein were more frequently noted in patients who died. In contrast, E. coli bacteremia, bacteremia due to urinary tract infections, biliary tract infections, or liver abscess, or underlying hypertension, were more often present in survivors at 28 days after bacteremia onset.

\section{Bacteremia isolates and susceptibility}

Because of 240 (10.2\%) events of polymicrobial bacteremia and 2109 monomicrobial bacteremia, a total of 2655 causative microorganisms from 2349 patients were collected. The ten leading microorganisms included E. coli $(968,36.5 \%)$, Klebsiella species (371, 14.0\%), Staphylococcus aureus (312, 11.8\%), Streptococcus species $(289,10.9 \%)$, Pseudomonas species (89, $3.4 \%)$, Enterococcus species (88, 3.3\%), Proteus species (68, 2.6\%), Salmonella species (59, 2.2\%), Aeromonas species (45, 1.7\%), and Enterobacter species (39, 1.5\%). Overall, cefazolin or cefuroxime was active against 7.6$78.0 \%$ or $15.4-89.3 \%$ of varied Gram-negative aerobes, respectively. Levofloxacin, cefotaxime, ceftazidime, cefepime, or ertapenem was active against $84.3-100 \%$, $86.1-96.6 \%$, $86.1-96.6 \%$, $94.4-100 \%$, or $96.8-100 \%$, respectively, of Gram-negative species, and imipenem was active against all Gram-negative aerobes. ESBL production was detected in 5.6\% (79) of 1401 EKP isolates. Of $312 \mathrm{~S}$. aureus isolates, $37.2 \%$ (116 isolates) were resistant to methicillin. 
Table 1 Patient demography, bacteremia severity, comorbidity severity, causative microorganisms, and laboratory data for adults with community-onset bacteremia, categorized by 28-day crude mortality

\begin{tabular}{|c|c|c|c|}
\hline \multirow[t]{2}{*}{ Variable } & \multicolumn{2}{|c|}{ 28-day Mortality, number of cases (\%) } & \multirow[t]{2}{*}{$P$ value } \\
\hline & Yes $(n=340)$ & No $(n=2009)$ & \\
\hline Age $\geq 65$ years & $223(65.6)$ & $1,227(61.1)$ & 0.11 \\
\hline Gender, male & $205(60.3)$ & $1,004(50.0)$ & $<0.001$ \\
\hline Nursing-home residents & $42(12.4)$ & $91(4.5)$ & $<0.001$ \\
\hline \multicolumn{4}{|l|}{ Severity-of-illness markers at arrival in the ED } \\
\hline Pitt bacteremia score $\geq 4$ & $217(63.8)$ & $259(12.9)$ & $<0.001$ \\
\hline \multicolumn{4}{|l|}{ Initial sepsis-related syndrome } \\
\hline Severe sepsis & $289(85.0)$ & $734(36.5)$ & $<0.001$ \\
\hline Septic shock & $225(66.2)$ & $254(12.6)$ & $<0.001$ \\
\hline Ultimately and rapidly fatal comorbidity (McCabe classification) & $159(46.8)$ & $405(20.2)$ & $<0.001$ \\
\hline Polymicrobial bacteremia & $62(18.2)$ & $178(8.9)$ & $<0.001$ \\
\hline \multicolumn{4}{|l|}{ Major causative microorganisms } \\
\hline Escherichia coli & $88(25.9)$ & $880(43.8)$ & $<0.001$ \\
\hline Klebsiella pneumoniae & $77(22.6)$ & $277(13.8)$ & $<0.001$ \\
\hline Staphylococcus aureus & $53(15.6)$ & $259(12.9)$ & 0.18 \\
\hline Streptococcus species & 45 (13.2) & $237(11.8)$ & 0.45 \\
\hline Pseudomonas species & $28(8.2)$ & $61(3.0)$ & $<0.001$ \\
\hline ESBL-producing EKP & $27(7.9)$ & $52(2.6)$ & $<0.001$ \\
\hline \multicolumn{4}{|l|}{ Major bacteremia sources } \\
\hline Pneumonia & $120(35.3)$ & $205(10.2)$ & $<0.001$ \\
\hline Urinary tract infection & 39 (11.5) & 709 (35.3) & $<0.001$ \\
\hline Biliary tract infection & $20(5.9)$ & $200(10.0)$ & 0.02 \\
\hline Intra-abdominal infection & $47(13.8)$ & $265(13.2)$ & 0.75 \\
\hline Liver abscess & $4(1.2)$ & $75(3.7)$ & 0.02 \\
\hline Skin and soft-tissue infection & $36(10.6)$ & $208(10.4)$ & 0.90 \\
\hline Primary bacteremia & $31(9.1)$ & $150(7.5)$ & 0.29 \\
\hline \multicolumn{4}{|l|}{ Major comorbidities } \\
\hline Malignancy & $159(46.8)$ & $500(24.9)$ & $<0.001$ \\
\hline Hypertension & $140(41.2)$ & $978(48.7)$ & 0.01 \\
\hline Diabetes mellitus & $117(34.4)$ & $758(37.7)$ & 0.24 \\
\hline Chronic kidney disease & $58(17.1)$ & $361(18.0)$ & 0.69 \\
\hline Neurological disease & $95(27.9)$ & $427(21.3)$ & 0.006 \\
\hline Liver cirrhosis & $70(20.6)$ & $231(11.5)$ & $<0.001$ \\
\hline \multicolumn{4}{|l|}{ Laboratory data on arrival at the ED } \\
\hline Leukocytes $\left(10^{3} / \mathrm{mm}^{3}\right)$ & $14.8 \pm 25.7$ & $12.5 \pm 7.2$ & 0.11 \\
\hline Platelet $\left(10^{3} / \mathrm{mm}^{3}\right)$ & $162.4 \pm 123.1$ & $199.4 \pm 114.4$ & $<0.001$ \\
\hline Serum creatinine (mg/dL) & $2.4 \pm 3.0$ & $2.2 \pm 15.3$ & 0.76 \\
\hline C-reactive protein (mg/L) & $162.3 \pm 147.5$ & $113.8 \pm 118.9$ & $<0.001$ \\
\hline
\end{tabular}

ED emergency department, ESBL extended-spectrum beta-lactamase, EKP Escherichia coli, Klebsiella species, and Proteus mirabilis

All variables are expressed as number of cases (\%), but laboratory data as mean \pm standard deviation

\section{Predictors of 28-day mortality}

The association between 28-day crude mortality and clinical variables, including age, sex, bacteremia severity, bacteremia source, comorbidity severity, causative microorganisms, and major comorbidities was examined by univariate analysis of 2349 adults. The following variables were positively associated with 28-day mortality: having male gender (odds ratio (OR) 1.52; $P<0.001$ ), being resident in a nursing home 
(OR 2.97; $P<0.001$ ), having ultimately or rapidly fatal comorbidities (McCabe classification; OR 3.48; $P<0.001$ ), being critically ill (PBS $\geq 4$; OR $11.92 ; P<0.001$ ) on arrival at the ED, having polymicrobial bacteremia (OR 2.29; $P<0.001$ ), K. pneumoniae (OR 1.83; $P<0.001$ ), Pseudomonas species (OR 2.87; $P<0.001$ ), or ESBL-producing EKP (OR 3.25; $P<0.001$ ) bacteremia, having bacteremia pneumonia (OR 4.80; $P<0.001$ ), and having comorbidities with malignancies (OR 2.65; $P<0.001$ ), neurological diseases (OR 2.00; $P=0.006$ ), or liver cirrhosis (OR 1.44; $P<0.001)$. Furthermore, the following variables were negatively associated with 28-day mortality: being mildly ill (PBS =0; OR 0.23; $P<0.001$ ), having $E$. coli bacteremia (OR $0.45 ; P<0.001$ ), having bacteremia due to urinary tract infections (OR $0.24 ; P<0.001$ ), having biliary tract infection (OR, 0.57; $P=0.02$ ) or liver abscess (OR 0.31; $P=0.02$ ), and having comorbidities with hypertension (OR $0.74 ; P=0.01$ ). However, eight independent predictors were identified in the multivariate regression analysis (Table 2): being critically ill (PBS $\geq 4$ ) on arrival at the ED, having ultimately or rapidly fatal comorbidities (McCabe classification), polymicrobial bacteremia, ESBL-producing EKP bacteremia, or bacteremia pneumonia, and preexisting malignancy or liver cirrhosis. One negative predictor was the presence of urinary tract infection as the source of bacteremia.

\section{Impact of different TtAa cutoffs}

Of 2349 patients with community-onset bacteremia, the mean (interquartile range) TtAa was $2.0(<1$ to 12$)$ hours. The majority (1663 patients, $70.8 \%)$ were treated by appropriate antimicrobials within 1 hour of arrival at the ED (Table 3). All selected TtAa cutoffs, ranging from 1 hour to 96 hours, had prognostic significance $(P<$

Table 2 Independent predictors of 28-day mortality in 2349 adults with community-onset bacteremia

\begin{tabular}{lll}
\hline Variables & AOR $(95 \% \mathrm{Cl})$ & $P$ value \\
\hline $\begin{array}{l}\text { Critical illness (Pitt bacteremia score } \geq 4) \\
\text { on arrival at the ED }\end{array}$ & $8.77(6.43-11.97)$ & $<0.001$ \\
$\begin{array}{l}\text { Ultimately and rapidly fatal comorbidity } \\
\text { (McCabe classification) }\end{array}$ & $2.21(1.48-2.92)$ & $<0.001$ \\
Polymicrobial bacteremia & $2.00(1.35-2.97)$ & 0.001 \\
$\begin{array}{l}\text { ESBL-producing EKP bacteremia } \\
\text { Bacteremia source }\end{array}$ & $6.00(3.11-11.55)$ & $<0.001$ \\
$\quad$ & $2.08(1.48-2.92)$ & $<0.001$ \\
$\quad$ Pneumonia & $0.46(0.25-0.84)$ & 0.01 \\
Urinary tract infection & & \\
$\quad$ Malignancy & $1.81(1.30-2.50)$ & $<0.001$ \\
$\quad$ Liver cirrhosis & $3.04(1.19-3.97)$ & $<0.001$ \\
\hline
\end{tabular}

$A O R$ adjusted odds ratio, $\mathrm{Cl}$ confidence interval, $E D$ emergency department, ESBL extended-spectrum beta-lactamase, EKP Escherichia coli, Klebsiella species, and Proteus mirabilis
0.001). In other words, those receiving appropriate antibiotics within the cutoff had a lower mortality rate than those treated by appropriate antibiotics after the cutoff time. The associated AORs ranged from 0.54 to 0.65 . Of importance, the lowest AOR (0.54) was evidenced in the TaAa cutoff of 48 hours.

\section{Impact of different TtAa cutoffs on patients with different severity of bacteremia}

According to the severity of bacteremia on arrival at the ED, all patients were categorized into three groups: mildly ill ( $\mathrm{PBS}=0 ; 633$ patients, 26.9\%), moderately ill (PBS $=1-3 ; 1240$ patients, 52.8\%), and critically ill (PBS $\geq 4$; 476 patients, $20.2 \%$ ), as shown in Table 4 . In mildly ill patients, after adjustment of independent predictors of 28-day mortality, only the TtAa cutoff of 48 hours (AOR = $0.47 ; P=0.04$ ) was significantly associated with 28-day mortality (Fig. 1a). Similarly, in moderately ill patients, three TtAa cutoffs (1 hour, 24 hours, and 48 hours) were significantly related to 28-day mortality, and the lowest AOR (0.55) was noted for the TtAa cutoff of 48 hours (Fig. 1b). Focusing on critically ill patients, all TtAa cutoffs were linked to 28-day mortality and the lowest AOR (0.56) was identified for the TtAa cutoff of 1 hour (Fig. 1c).

\section{Discussion}

Bacteremia is associated with substantial morbidity and mortality and early interventions are often emphasized $[1,14]$. However, the estimates of potential benefits of appropriate empirical antibiotic treatment vary widely in the literature, from no effect $[8,9]$ to significant reduction in fatality with an odds ratio of up to 6 [4-7]. Such a controversy may be related to variations in sepsis severity, comorbidities and the immune status of study cohorts, and the distribution of causative microorganisms [24]. Similar to previous reports dealing with the crucial relationship between patient outcomes and the appropriateness of empirical antimicrobial therapy for specific pathogens, such as Gram-negative bacilli [25], E. coli [26, 27], K. pneumoniae [27], S. aureus [28], or bloodstream infections $[5,6]$, our results support that appropriate empirical antimicrobial therapy reduces short-term mortality in patients with community-onset bacteremia. Of importance, in accordance with increasing bacteremia severity, appropriate antimicrobials should be given as soon as possible.

Several investigations have attempted to answer the question "How early is enough to administer appropriate antimicrobials in patients with severe infection?" However, different TtAa cutoffs were determined in heterogeneous patient populations reported in the literature. First, for patients with severe Legionella pneumophila pneumonia, delays $>8$ hours between appropriate antimicrobial administration and ICU 
Table 3 Critical illness, fatal comorbidities, and 28-day mortality rates among adults with community-onset bacteremia

\begin{tabular}{|c|c|c|c|c|c|c|c|}
\hline \multirow{2}{*}{$\begin{array}{l}\text { TtAa cutoffs } \\
\text { (number of cases) }\end{array}$} & \multicolumn{2}{|c|}{ Percentage (number of cases) } & \multirow{2}{*}{$\begin{array}{l}\text { 28-day Mortality rate } \\
\text { (number of cases) }\end{array}$} & \multicolumn{2}{|c|}{ Univariate analysis } & \multicolumn{2}{|l|}{ Cox regression } \\
\hline & Critical illness $^{a}$ & Fatal comorbidity & & OR $(95 \% \mathrm{Cl})$ & $P$ value & $\overline{A O R}(95 \% \mathrm{Cl})^{\mathrm{b}}$ & $P$ value \\
\hline \multicolumn{8}{|l|}{1 hour } \\
\hline$\leq 1(n=1663)$ & $16.5(275)$ & $22.4(373)$ & $10.3(172)$ & $0.36(0.28-0.45)$ & $<0.001$ & $0.57(0.46-0.71)$ & $<0.001$ \\
\hline$>1(n=686)$ & $29.3(201)$ & $27.8(191)$ & $24.5(168)$ & & & & \\
\hline \multicolumn{8}{|l|}{6 hours } \\
\hline$\leq 6(n=1736)$ & $19.0(329)$ & $23.1(401)$ & $12.0(208)$ & $0.50(0.39-0.63)$ & $<0.001$ & $0.65(0.51-0.82)$ & $<0.001$ \\
\hline$>6(n=613)$ & $24.0(147)$ & $26.6(163)$ & $21.5(132)$ & & & & \\
\hline \multicolumn{8}{|l|}{12 hours } \\
\hline$\leq 12(n=1760)$ & $19.4(341)$ & $23.4(412)$ & $12.3(216)$ & $0.53(0.41-0.67)$ & $<0.001$ & $0.65(0.52-0.83)$ & $<0.001$ \\
\hline$>12(n=589)$ & $22.9(135)$ & $25.8(152)$ & $21.1(124)$ & & & & \\
\hline \multicolumn{8}{|l|}{24 hours } \\
\hline$\leq 24(n=1,853)$ & $19.8(367)$ & $23.5(436)$ & $12.6(234)$ & $0.53(0.41-0.69)$ & $<0.001$ & $0.62(0.48-0.79)$ & $<0.001$ \\
\hline$>24(n=496)$ & $22.0(109)$ & $25.8(128)$ & $21.4(106)$ & & & & \\
\hline \multicolumn{8}{|l|}{48 hours } \\
\hline$\leq 48(n=1,917)$ & $19.8(380)$ & $23.5(450)$ & $12.5(240)$ & $0.48(0.37-0.62)$ & $<0.001$ & $0.54(0.43-0.71)$ & $<0.001$ \\
\hline$>48(n=432)$ & $22.2(96)$ & $26.4(114)$ & $23.1(100)$ & & & & \\
\hline \multicolumn{8}{|l|}{72 hours } \\
\hline$\leq 72(n=1,981)$ & 19.5 (387) & 23.4 (463) & $12.6(249)$ & $0.44(0.33-0.57)$ & $<0.001$ & $0.55(0.43-0.71)$ & $<0.001$ \\
\hline$>72(n=368)$ & $24.2(89)$ & $27.4(101)$ & $24.7(91)$ & & & & \\
\hline \multicolumn{8}{|l|}{96 hours } \\
\hline$\leq 96(n=2,061)$ & $19.4(400)$ & $23.5(484)$ & $12.8(264)$ & $0.41(0.31-0.55)$ & $<0.001$ & $0.56(0.44-0.72)$ & $<0.001$ \\
\hline$>96(n=288)$ & $26.4(76)$ & $27.8(80)$ & $26.4(76)$ & & & & \\
\hline
\end{tabular}

The significance of the effects of different cutoffs of the time to appropriate antibiotic (TtAa) on 28-day mortality were examined by univariate and Cox regression analyses. OR odds ratio, $\mathrm{Cl}$ confidence interval, $A O R$ adjusted odds ratio. ${ }^{\mathrm{a}}$ Pitt bacteremia score $\geq 4$ on arrival at the emergency department. ${ }^{\mathrm{b}} \mathrm{Adjusted}$ for independent predictors of 28-day mortality recognized in the multivariate regression: critical illness; a fatal comorbidity (McCabe classification); polymicrobial bacteremia; Extendedspectrum beta-lactamase-producing bacteremia; bacteremia because of pneumonia or urinary tract infections; and underlying malignancy or liver cirrhosis

admission resulted in increased mortality [29]. Second, in another study of patients with cancer and septic shock, a delay of $>2$ hours from ICU admission was associated with a poor prognosis [30]. Finally, in a study of ED patients with severe sepsis and septic shock, a TtAa $<1$ hour led to a better short-term survival rate [18].

To our knowledge, the present study was the first one focusing on community-onset bacteremia to prioritize antimicrobial therapy in the initial therapeutic strategy. Similar to a previous report of hospital-based bloodstream infection [7], the "optimum timing" of appropriate antimicrobial administration was determined as the first 48 hours after arrival at the ED. Furthermore, consistent with the SSC recommendations [3], this time should be shortened to 1 hour in critically ill patients. However, the administration of in vitro effective antimicrobials to treat bloodstream infections should be as early as possible to achieve optimal treatment of bacterial infections. Any delay in the administration of appropriate drugs should be avoided, if there is a suspicion of severe sepsis or septic shock.
This study has several limitations. First, antimicrobial therapy is one of the cornerstones of therapeutic strategies for patients with bloodstream infection. Nevertheless, for critically ill patients with the complications of severe sepsis and septic shock, other elements of early goal-directed therapy, such as central venous pressure, mean arterial pressure, and central venous oxygen saturation, were not evaluated as covariates in this study. Second, in the assessment of the primary endpoint and analysis of the impact of antimicrobial therapy, we excluded patients with incomplete clinical information and those receiving inappropriate antibiotic dosage or route of administration. As a result, only 16 patients were excluded and thus the selection bias was expected to be minimal. Third, as previous investigations dealing with the effects of the appropriateness of empirical antimicrobial administration on patient outcome [4-9], no detailed information was available in our study on early resuscitation. However, the majority $(1663,70.8 \%)$ of the 2349 patients with community-onset bacteremia received the first dose of appropriate antimicrobials within one hour of arrival at the ED, and the study population 
Table 4 Impact of different cutoffs for the time-to-appropriate antibiotic (TtAa) on 28-day crude mortality in adults categorized by Pitt bacteremia score (PBS) on arrival at the emergency department

\begin{tabular}{|c|c|c|c|c|}
\hline \multirow{2}{*}{$\begin{array}{l}\text { TtAa } \\
\text { (hours) }\end{array}$} & \multicolumn{2}{|c|}{ Mortality rate (\%) } & \multicolumn{2}{|c|}{ Univariate analysis } \\
\hline & $\leq \mathrm{TtAa}$ & $>\mathrm{TtAa}$ & OR $(95 \% \mathrm{Cl})$ & $P$ value \\
\hline \multicolumn{5}{|c|}{ Mildly ill (PBS $=0, n=633)$} \\
\hline 1 & 3.6 & 8.4 & $0.41(0.20-0.85)$ & 0.01 \\
\hline 6 & 3.6 & 8.8 & $0.39(0.19-0.80)$ & 0.008 \\
\hline 12 & 3.8 & 8.3 & $0.43(0.21-0.90)$ & 0.02 \\
\hline 24 & 3.7 & 9.1 & $0.38(0.18-0.80)$ & 0.008 \\
\hline 48 & 3.6 & 10.2 & $0.33(0.16-0.69)$ & 0.002 \\
\hline 72 & 3.8 & 10.5 & $0.34(0.16-0.73)$ & 0.004 \\
\hline 96 & 4.4 & 8.5 & $0.48(0.20-1.17)$ & 0.10 \\
\hline
\end{tabular}

Moderately ill (PSB $=1-3, n=1240$ )

$\begin{array}{lllll}1 & 6.0 & 11.6 & 0.48(0.31-0.75) & 0.001 \\ 6 & 6.3 & 10.7 & 0.56(0.36-0.88) & 0.01 \\ 12 & 6.3 & 11.1 & 0.54(0.34-0.84) & 0.006 \\ 24 & 6.3 & 11.9 & 0.50(0.32-0.80) & 0.003 \\ 48 & 6.3 & 13.0 & 0.45(0.28-0.73) & 0.001 \\ 72 & 6.6 & 12.6 & 0.49(0.29-0.81) & 0.005 \\ 96 & 6.8 & 13.1 & 0.48(0.28-0.84) & 0.009\end{array}$

Critical ill (PBS $\geq 4, n=476$ )

\begin{tabular}{lllll}
1 & 36.4 & 58.2 & $0.41(0.28-0.60)$ & $<0.001$ \\
6 & 40.1 & 57.8 & $0.49(0.33-0.73)$ & $<0.001$ \\
12 & 40.8 & 57.8 & $0.50(0.34-0.75)$ & 0.001 \\
24 & 41.7 & 58.7 & $0.50(0.33-0.78)$ & 0.002 \\
48 & 41.3 & 62.5 & $0.42(0.27-0.67)$ & $<0.001$ \\
72 & 41.1 & 62.4 & $0.43(0.23-0.60)$ & $<0.001$ \\
96 & 41.2 & 62.5 & $0.43(0.24-0.62)$ & $<0.001$ \\
\hline
\end{tabular}

OR odds ratio, $\mathrm{Cl}$ confidence interval

were believed to be managed according to the SSC recommendations. Thus, the clinical outcomes of the patients receiving appropriate antimicrobial therapy at different time points after arrival at the ED could be compared with each other. Fourth, as noted before [49], no analyses of source control were performed in the present study. However, taking the cases of urosepsis as the example, in those with or without interventions for source control, such as percutaneous nephrolithotomy or nephrectomy, the 28-day crude mortality rate was not different $(4 / 48,8.3 \%$ vs. $35 / 700,5.0 \% ; P=0.31)$, indicative of standard quality of clinical care in the study hospital. Finally, as this study was conducted at one hospital, the critical TtAa cutoffs we identified may not be generalizable to other communities with variable infection sources or severity of community-onset bacteremia. However, our findings highlight that more severely ill patients with bacteremia require earlier

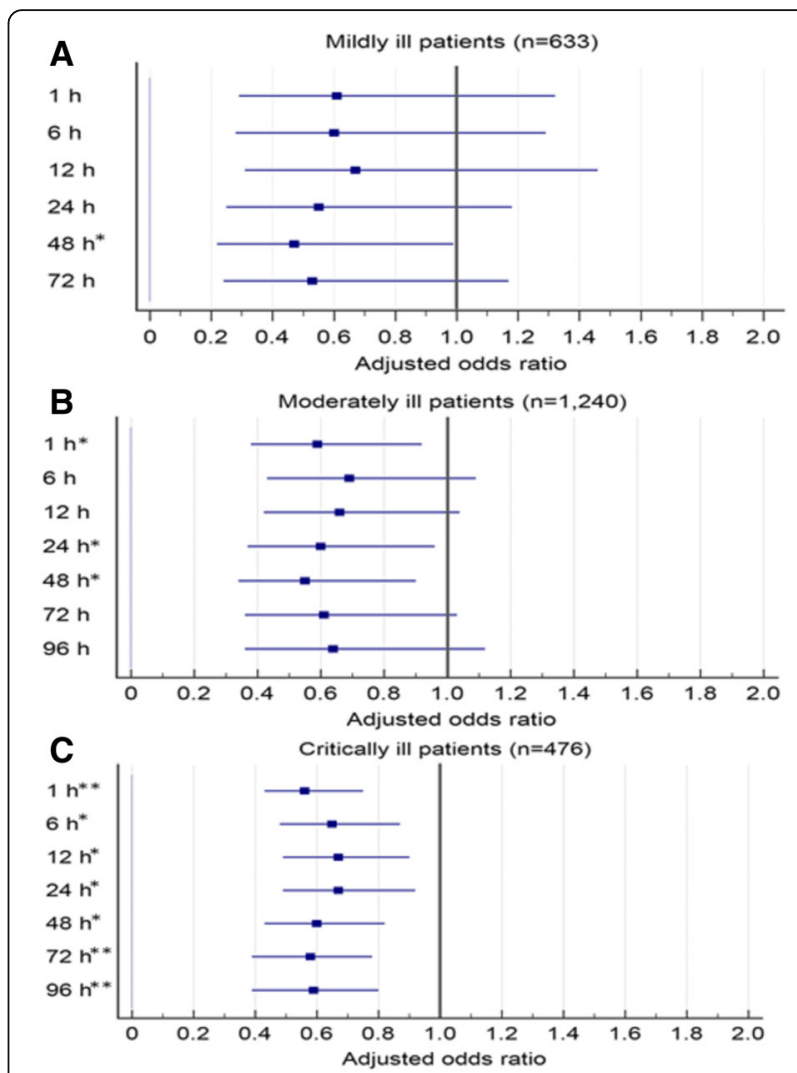

Fig. 1 Adjusted odds ratios of different cutoffs of the time-toappropriate antibiotic for 28-day crude mortality in adults, categorized by the Pitt bacteremia score on arrival at the emergency department in mildly ill (a), moderately ill (b), and critically ill (c) patients, using Cox regression after adjustment for the independent predictors of 28-day mortality (including a fatal comorbidity (McCabe classification), polymicrobial bacteremia, extended-spectrum beta-lactamaseproducing bacteremia, bacteremia because of pneumonia or urinary tract infections, and underlying malignancies or liver cirrhosis). ${ }^{*} P$ value $<0.05$; ${ }^{* *} P$ value $<0.001$

administration of appropriate antimicrobials to achieve a favorable outcome.

\section{Conclusions}

Our results demonstrate that the elapsed time from arrival at the ED to the administration of appropriate antimicrobials is a crucial determinant of short-term outcomes in patients with community-onset bacteremia. The optimum timing of appropriate antimicrobial administration in mildly ill patients was the first 48 hours after arrival at the ED. With increasing severity of bacteremia, this time should be limited to the first 1 hour in critically ill patients. Therefore, to achieve a favorable outcome in critically ill patients, epidemiological surveillance, rapid pathogen identification, or the incorporation of broadspectrum antimicrobials as empirical therapy into an antibiotic stewardship program should be considered. 


\section{Abbreviations}

AOR: Adjusted odds ratio; Cl: Confidence interval; CLSI: Clinical and Laboratory Standards Institute; ED: Emergency department; EKP: Escherichia coli, Klebsiella species, and Proteus mirabilis; ESBL: Extended-spectrum betalactamase; ICUs: Intensive care units; OR: Odds ratio; PBS: Pitt bacteremia score; SSC: Surviving Sepsis Campaign; TtAa: Time to appropriate antibiotic

\section{Acknowledgements}

We would like to thank all the anonymous reviewers for their valuable comments and suggestions on improving the quality of our study.

\section{Funding}

This study was partially supported by research grants from the Ministry of Science and Technology (NSC102-2314-B-006-079), Ministry of Health and Welfare (MOHW106-TDU-B-211-113003) and National Cheng Kung University Hospital (NCKUH-10305018 and NCKUH-10406029), Tainan, Taiwan.

\section{Availability of data and materials}

All data are fully available without restriction.

\section{Authors' contributions}

$\mathrm{CL}, \mathrm{WK}$, and $\mathrm{HT}$ conceived the study idea and designed the study. CL, HL, and $\mathrm{MH}$ supervised the data collection and chart reviews. CL, HL, WK, and HT provided methodological and statistical advice on study design and data analysis. CL, WK, and HT provided expertise in infectious disease, helped to draft this manuscript, and revised it carefully from a professional point of view. All authors read and approved the final manuscript.

\section{Competing interests}

The authors declare that they have no competing interests.

\section{Consent for publication}

All authors have provided consent for publication of the manuscript.

\section{Ethics approval and consent to participate}

The study was approved by the institutional review board of National Cheng Kung University Hospital (ER-100-182), and the requirement of obtaining informed consent was waived.

\section{Publisher's Note}

Springer Nature remains neutral with regard to jurisdictional claims in published maps and institutional affiliations.

\section{Author details}

${ }^{1}$ Division of Critical Care Medicine, Department of Internal Medicine, Madou Sin-Lau Hospital, No. 20, Lingzilin, 72152, Madou Dist, Tainan City, Taiwan. ${ }^{2}$ Graduate Institute of Medical Sciences, College of Health Sciences, Chang Jung Christian University, Tainan, Taiwan. ${ }^{3}$ Department of Internal Medicine, National Cheng Kung University Hospital, Tainan, Taiwan. ${ }^{4}$ Department of Medicine, National Cheng Kung University Medical College, Tainan, Taiwan. ${ }^{5}$ Department of Emergency Medicine, National Cheng Kung University Hospital, No. 138, Sheng Li Road, 70403 Tainan, Taiwan. ${ }^{6}$ Department of Medicine, Chi-Mei Medical Center, Tainan, Taiwan. ${ }^{7}$ Department of Health and Nutrition, Chia Nan University of Pharmacy and Science, Tainan, Taiwan. ${ }^{8}$ Division of Infectious Disease, Department of Internal Medicine, National Cheng Kung University Hospital, No. 138, Sheng Li Road, 70403 Tainan, Taiwan. ${ }^{9}$ Division of Infectious Disease, Department of Medicine, Chi-Mei Medical Center, No. 901, Chung-Hwa Road, Yung-Kang City 710, Tainan, Taiwan.

\section{Received: 21 January 2017 Accepted: 2 May 2017} Published online: 26 May 2017

\section{References}

1. Bates DW, Pruess KE, Lee TH. How bad are bacteremia and sepsis? Outcomes in a cohort with suspected bacteremia. Arch Intern Med. 1995; 155(6):593-8.

2. Laupland KB, Gregson DB, Flemons WW, Hawkins D, Ross T, Church DL. Burden of community-onset bloodstream infection: a population-based assessment. Epidemiol Infect. 2007;135(6):1037-42.
3. Rhodes A, Evans LE, Alhazzani W, Levy MM, Antonelli M, Ferrer R, et al. Surviving Sepsis Campaign: international guidelines for management of sepsis and septic shock: 2016. Crit Care Med. 2017;45(3):1-67.

4. Ibrahim EH, Sherman G, Ward S, Fraser VJ, Kollef MH. The influence of inadequate antimicrobial treatment of bloodstream infections on patient outcomes in the ICU setting. Chest. 2000;118(1):146-55.

5. Lee CC, Lee CH, Chuang MC, Hong MY, Hsu HC, Ko WC. Impact of inappropriate empirical antibiotic therapy on outcome of bacteremic adults visiting the ED. Am J Emerg Med. 2012;30(8):1447-56.

6. Chen HC, Lin WL, Lin CC, Hsieh WH, Hsieh CH, Wu MH, et al. Outcome of inadequate empirical antibiotic therapy in emergency department patients with community-onset bloodstream infections. J Antimicrob Chemother. 2013:68(4):947-53

7. Leibovici L, Shraga I, Drucker M, Konigsberger H, Samra Z, Pitlik SD. The benefit of appropriate empirical antibiotic treatment in patients with bloodstream infection. J Intern Med. 1998;244(5):379-86.

8. Lin MY, Weinstein RA, Hota B. Delay of active antimicrobial therapy and mortality among patients with bacteremia: impact of severe neutropenia. Antimicrob Agents Chemother. 2008;52(9):3188-94.

9. Corona A, Bertolini G, Lipman J, Wilson AP, Singer M. Antibiotic use and impact on outcome from bacteraemic critical illness: the BActeraemia Study in Intensive Care (BASIC). J Antimicrob Chemother. 2010;65(6):1276-85.

10. Hsieh CC, Lee CH, Hong MY, Hung YP, Lee NY, Ko WC, et al. Propensity score-matched analysis comparing the therapeutic efficacies of cefazolin and extended-spectrum cephalosporins as appropriate empirical therapy in adults with community-onset Escherichia coli, Klebsiella spp. and Proteus mirabilis bacteraemia. Int J Antimicrob Agents. 2016;48(6):712-8.

11. Lee CC, Wang JL, Lee CH, Hsieh CC, Hung YP, Hong MY, et al. Clinical benefit of appropriate empirical fluoroquinolone therapy for adults with community-onset bacteremia in comparison with third-generationcephalosporin therapy. Antimicrob Agents Chemother. 2017;61(2): e02174-16.

12. Hsieh CC, Lee CH, Li MC, Hong MY, Chi CH, Lee CC. Empirical thirdgeneration cephalosporin therapy for adults with community-onset Enterobacteriaceae bacteraemia: impact of revised CLSI breakpoints. Int J Antimicrob Agents. 2016:47(4):297-303.

13. Lee $\mathrm{CH}$, Hsieh CC, Hong MY, Hung YP, Ko WC, Lee CC. Comparing the therapeutic efficacies of third-generation cephalosporins and broaderspectrum beta-lactams as appropriate empirical therapy in adults with community-onset monomicrobial Enterobacteriaceae bacteraemia: a propensity-score matched analysis. Int J Antimicrob Agents. 2017;49(5): 617-23.

14. Lee CC, Lee NY, Chen PL, Hong MY, Chan TY, Chi CH, et al. Impact of antimicrobial strategies on clinical outcomes of adults with septic shock and community-onset Enterobacteriaceae bacteremia: de-escalation is beneficial. Diagn Microbiol Infect Dis. 2015;82(2):158-64.

15. Lee CC, Lin WJ, Shih HI, Wu CJ, Chen PL, Lee HC, et al. Clinical significance of potential contaminants in blood cultures among patients in a medical center. J Microbiol Immunol Infect. 2007:40(5):438-44.

16. Gilbert DN, Moellering RC Jr., Eliopoulos GM, ChambersHF, Saag MS. The Sanford guide to antimicrobial therapy. 39th ed. Sperryville, VA: Antimicrobial Therapy. 2009;78-82.

17. Clinical and Laboratory Standards Institute. Performance standards for antimicrobial susceptibility testing; approved standard. Twenty-six informational supplement. CLSI document M100-S26. Wayne, PA: CLSI; 2016.

18. Gaieski DF, Mikkelsen ME, Band RA, Pines JM, Massone R, Furia FF, et al. Impact of time to antibiotics on survival in patients with severe sepsis or septic shock in whom early goal-directed therapy was initiated in the emergency department. Crit Care Med. 2010;38(4):1045-53.

19. Schellevis FG, van der Velden J, van de Lisdonk E, van Eijk JT, van Weel C. Comorbidity of chronic diseases in general practice. J Clin Epidemiol. 1993; 46(5):469-73.

20. McCabe WR. Gram-negative bacteremia. Adv Intern Med. 1974;19:135-58.

21. Clinical and Laboratory Standards Institute. Performance standards for antimicrobial susceptibility testing; approved standard. Nineteenth informational supplement. CLSI document M100-S19. Wayne, PA: CLSI; 2009.

22. Peduzzi P, Concato J, Kemper E, Holford TR, Feinstein AR. A simulation study of the number of events per variable in logistic regression analysis. J Clin Epidemiol. 1996;49(12):1373-9.

23. Royston P, Moons KG, Altman DG, Vergouwe Y. Prognosis and prognostic research: developing a prognostic model. BMJ. 2009;338:b604. 
24. Kumar A. An alternate pathophysiologic paradigm of sepsis and septic shock: implications for optimizing antimicrobial therapy. Virulence. 2014;5(1):80-97.

25. Bryan CS, Reynolds KL, Brenner ER. Analysis of 1,186 episodes of gramnegative bacteremia in non-university hospitals: the effects of antimicrobial therapy. Rev Infect Dis. 1983;5(4):629-38.

26. Peralta G, Sanchez MB, Garrido JC, De Benito I, Cano ME, Martinez-Martinez $L$, et al. Impact of antibiotic resistance and of adequate empirical antibiotic treatment in the prognosis of patients with Escherichia coli bacteraemia. J Antimicrob Chemother. 2007;60(4):855-63.

27. Lautenbach E, Metlay JP, Bilker WB, Edelstein PH, Fishman NO. Association between fluoroquinolone resistance and mortality in Escherichia coli and Klebsiella pneumoniae infections: the role of inadequate empirical antimicrobial therapy. Clin Infect Dis. 2005:41(7):923-9.

28. Khatib R, Saeed S, Sharma M, Riederer K, Fakih MG, Johnson LB. Impact of initial antibiotic choice and delayed appropriate treatment on the outcome of Staphylococcus aureus bacteremia. Eur J Clin Microbiol Infect Dis. 2006; 25(3):181-5.

29. Gacouin A, Le Tulzo Y, Lavoue S, Camus C, Hoff J, Bassen R, et al. Severe pneumonia due to Legionella pneumophila: prognostic factors, impact of delayed appropriate antimicrobial therapy. Intensive Care Med. 2002;28(6):686-91.

30. Larche J, Azoulay E, Fieux F, Mesnard L, Moreau D, Thiery G, et al. Improved survival of critically ill cancer patients with septic shock. Intensive Care Med. 2003;29(10):1688-95

\section{Submit your next manuscript to BioMed Central and we will help you at every step:}

- We accept pre-submission inquiries

- Our selector tool helps you to find the most relevant journal

- We provide round the clock customer support

- Convenient online submission

- Thorough peer review

- Inclusion in PubMed and all major indexing services

- Maximum visibility for your research

Submit your manuscript at www.biomedcentral.com/submit 